\title{
ON FINITELY GENERATED SUBGROUPS OF FREE PRODUCTS
}

\author{
R. G. BURNS \\ (Received 15 April 1969; revised 6 August 1969) \\ Communicated by B. Mond
}

\section{Statement of Results}

If $H$ is a subgroup of a group $G$ we shall say that $G$ is $H$-residually finite if for every element $g$ in $G$, outside $H$, there is a subgroup of finite index in $G$, containing $H$ and still avoiding $g$. (Then, according to the usual definition, $G$ is residually finite if it is $E$-residually finite, where $E$ is the identity subgroup). Definitions of other terms used below may be found in $\S 2$ or in [6].

In this note we obtain the following result, proved in a slightly more general form as Theorem 3.1.

1.1 Theorem. Suppose $G$ is the free product of its subgroups $A_{i}$ indexed by some set $I$, and let $H$ be a finitely generated subgroup. The following two conclusions hold.

1.1.1 If for each $i \in I, g \in G, A_{i}$ is $\left(g^{-1} H g \cap A_{i}\right)$-residually finite, then $G$ is $H$-residually finite.

1.1.2 If the $A_{i}$ are residually finite and if for each $i \in I, g \in G, g^{-1} \mathrm{Hg} \cap A_{i}$ is a free factor of a subgroup of finite index in $A_{i}$, then $H$ is a free factor of a subgroup of finite index in $G$.

This theorem and Theorem 3.1 generalize Theorem 1 of [1], and the idea of the proof is the same.

Statement 1.1.1 is a generalization both of the result of M. Hall, Jr. [4] that a free group is $H$-residually finite for all finitely generated subgroups $H$, and of the result (Gruenberg [3]) that a free product of residually finite groups is residually finite. Statement 1.1.2 generalizes the result ([1]) that a finitely generated subgroup of a free group is a free factor of a subgroup of finite index. C.f. also [7].

We make a few further brief observations. The converse of 1.1.2 is true without the condition that the $A_{i}$ be residually finite. (This is a simple consequence of the Kurosh subgroup theorem (see $\S 2$, Theorem 2.3). A counterexample to show that 1.1.2 is not true without some such hypothesis is provided by the free product of a 2-cycle and the Prüfer group $C_{2} \infty$, taking for $H$ the infinite cycle generated by any element of length 2 . 
Secondly we observe that if we combine the hypotheses of both 1.1.1 and 1.1.2, then given $g \in G \backslash H$, there is a subgroup of finite index in $G$, containing $H$ as a free factor and avoiding $g$ : i.e. a subgroup satisfying simultaneously the conclusions of 1.1.1 and 1.1.2 can be found. This follows from Lemma 2.5 below.

Finally, if (following a suggestion of S. Meskin) we define a group to be extended residually finite ${ }^{1}$ if it is $H$-residually finite for all subgroups $H$, and locally-extended residually finite if it is $H$-residually finite for all finitely generated subgroups $H$, then we have the following result as a simple consequence of 1.1.1 and the Kurosh subgroup theorem.

1.2 COROLLARY. The class of locally-extended residually finite groups is closed under formation of free products.

If we denote by $\mathscr{F}$ the class of finite groups, and by $R \mathscr{F}, E R \mathscr{F}$ and $L E R \mathscr{F}$ the classes of residually finite, extended residually finite and locally-extended residually finite groups respectively, it is not difficult to see that

$$
\mathscr{F} \subset E R \mathscr{F} \subset L E R \mathscr{F} \subset R \mathscr{F},
$$

where $\subset$ denotes strict inclusion. Thus by Corollary 1.2 and Gruenberg's result respectively, LER $\mathscr{F}$ and $R \mathscr{F}$ are closed under free product formation. On the other hand $\mathscr{F}$ is trivially not, and, since free groups of rank $>1$ do not belong to $E R \mathscr{F}$, neither is $E R \mathscr{F}$.

I thank Drs. S. Meskin and R. Gregorac for helpful comments.

\section{Preliminaries}

The following more-or-less well-known definitions and results are needed for the proof of our Theorem 3.1. For the sake of precision we include the definition of a free product. The identity will be denoted throughout by $e$.

2.1 Definition. Let $G$ be a group and $\left\{A_{i} \mid i \in I\right\}$ a set of subgroups indexed by $I$. We say that $G$ is a free product of the $A_{i}$ if every non-trivial element $g \in G$ can be written uniquely in the form $a_{i_{1}} \cdots a_{i_{n}}$ where $e \neq a_{i_{k}} \in A_{i_{k}}(k=1, \cdots, n)$ and $i_{k} \neq i_{k+1}(k=1, \cdots, n-1)$. We say that this is the reduced form of $g$, that $g$ has length $n$ (ascribing length zero to $e$ ), and that $g$ ends in $a_{i_{n}}$ (and $e$ ends in no element). The $A_{i}$ are called free factors of $G$ and we write $G=\prod_{i \in I}^{*} A_{i}$, or briefly $G=\prod^{*} A_{i}$.

Note that every group has at least two free factors, namely itself and $E$.

In order to state the Kurosh subgroup theorem and a converse of it due to Dey [2], we need the concept of a uniform Schreier system in a free product. It is straightforward to verify that the following definition is interchangeable with that of Dey [2, Definition 2.1] or that on p. 239 of [6], except that ours includes im-

${ }^{1}$ Termed 'a group with finitely distinguishable subgroups' by A. I. Mal'cev. (On homomorphisms onto finite groups, Ivanov. Gos. Ped. Inst. Učen. Zap., 18 (1958), 49-60.) 
plicitly the specification of 'a set of admissible functions' (see [2]). The following formulation is convenient for the proof of Theorem 3.1.

2.2 Definition. Let $G$ be a free product $\Pi^{*} A_{i}$. A uniform Schreier system is a triple

$$
\text { 2.2.1 }\left(\left\{T_{i}, S_{i} \mid i \in I\right\},\left\{B(i, \sigma), Z(i, \sigma) \mid i \in I, \sigma \in S_{i}\right\},\left\{\theta_{i j} \mid i, j \in I\right\}\right)
$$

with the following properties:

2.2.2 For all $i \in I, T_{i} \subseteq G, e \in T_{i}$; if $t \in \bigcup_{i \in I} T_{1}$ ends in an element $a_{j} \in A_{j}$, then $t, t a_{j}^{-1} \in T_{j}$.

2.2.3 For all $i \in I, S_{i}$ is the subset of those elements of $T_{i}$ which do not end in an element of $A_{i}$; for each pair $(i, \sigma), i \in I, \sigma \in S_{i}, B(i, \sigma)$ is a subgroup of $A_{i}$, and $Z(i, \sigma)$ is a right transversal for $B(i, \sigma)$ in $A_{i}$ such that

$$
\sigma Z(i, \sigma)=T_{i} \cap \sigma A_{i} \text {. }
$$

2.2.4 For each ordered pair $(i, j) \in I \times I, \theta_{i j}: T_{i} \rightarrow T_{j}$ is a bijection satisfying: (i) $\theta_{i j}=\theta_{j i}^{-1}$; (ii) $\theta_{i j}$ is the identity map on $T_{i} \cap T_{j}$; (iii) $\theta_{i k} \theta_{k j}=\theta_{i j}$ for all $k \in I$.

In the following statements we include explicitly only those details relevant to the present note.

2.3 THEOREM. (Kurosh) (cf. [5]) If $G=\prod^{*} A_{i}$ and $H$ is a subgroup of $G$ (briefly $H \leqq G$ ) then there exists a uniform Schreier system 2.2.1 such that

where $F$ is free on the set

$$
H=F * \prod_{i \in I}^{*} \prod_{\sigma \in S_{i}}^{*} \sigma B(i, \sigma) \sigma^{-1}
$$

$$
\left\{t_{i}\left(t_{i} \theta_{i \alpha}\right)^{-1} \mid \alpha, i \in I, \alpha \text { fixed, } t_{i} \in T_{i}, t_{i}\left(t_{i} \theta_{i \alpha}\right)^{-1} \neq e\right\} .
$$

In addition it follows that for each $(i, \sigma), i \in I, \sigma \in S_{i}$,

2.3.1 $B(i, \sigma)=\sigma^{-1} H \sigma \cap A_{i}$.

2.4 TheOREM. (Dey [2, Theorem 3.11]) Given $G=\prod^{*} A_{i}$ and any uniform Schreier system 2.2.1 in $G$, then the set

$$
\left\{t_{i}\left(t_{i} \theta_{i \alpha}\right)^{-1} \mid \alpha, i \in I, \alpha \text { fixed, } t_{i} \in T_{i}, t_{i}\left(t_{i} \theta_{i \alpha}\right)^{-1} \neq e\right\}
$$

freely generates a free group $F_{1}$, say, and the subgroup closure $H_{1}$ of $F_{1}$ and the subgroups $\sigma B(i, \sigma) \sigma^{-1}, i \in I, \sigma \in S_{i}$, is the free product of $F$ and these subgroups. It then also follows that for each $i, T_{i}$ is a right transversal for $H_{1}$ in $G$.

Lastly we state the following lemma.

2.5 Lemma. If a group $G$ is $H$-residually finite for some subgroup $H$ which is also a free factor of a subgroup of finite index in $G$, then given any finite subset $S \subseteq G \backslash H$, there is a subgroup of finite index in $G$, containing $H$ as a free factor and avoiding $S$ (i.e. there is a subgroup serving both purposes at once). 
The proof is trivial once the following simple corollary of the Kurosh subgroup theorem (2.3) is recalled:

If $A$ is a free factor of a group $G$ and $B \leqq G$, then $A \cap B$ is a free factor of $B$.

\section{The Theorem}

3.1 TheORem. Let $H$ be a subgroup of a free product $G=\prod^{*} A_{i}$, with a corresponding uniform Schreier system

$$
\left.\left(\left\{T_{i}, S_{i}\right\} \mid i \in I\right\},\left\{B(i, \sigma), Z(i, \sigma) \mid i \in I, \sigma \in S_{i}\right\},\left\{\theta_{i j} \mid i, j \in I\right\}\right)
$$

yielding the free decomposition

$$
H=F * \prod_{i \in I}^{*} \prod_{\sigma \in S_{i}}^{*} \sigma B(i, \sigma) \sigma^{-1}
$$

in accordance with Theorem 2.3, such that $F$ has finite rank and the set

$$
Q=\left\{(i, \sigma) \mid i \in I, \sigma \in S_{i}, B(i, \sigma) \neq E\right\}
$$

is finite. Suppose further that for all $i \in I, g \in G, A_{i}$ is $\left(g^{-1} H g \cap A_{i}\right)$-residually finite. Then $G$ is $H$-residually finite.

If in addition to the above assumptions on $H$, for all $i \in I, g \in G, g^{-1} H g \cap A_{i}$ is a free factor of a subgroup of $A_{i}$ of finite index, then $H$ is a free factor of a subgroup of $G$ of finite index.

Statement 1.1.1 of Theorem 1.1 follows immediately and 1.1.2 is also easily deduced once the following fact is noted:

If $K$ is a free factor of a subgroup $B$ of finite index in a group $A\left(B=K * K_{1}\right.$ say) and $K_{1}$ is residually finite, then $A$ is $K$-residually finite.

The proof of this is as follows. By Theorem 3.1, $B$ is $K$-residually finite. It is then a straightforward consequence of the definition that since $B$ has finite index in $A$, the latter is also $K$-residually finite.

Proof OF 3.1. By the Kurosh subgroup theorem (2.3) $F$ is freely generated by the set

Define

$$
\left\{t_{i}\left(t_{i} \theta_{i \alpha}\right)^{-1} \mid \alpha, i \in I\right\} \alpha \text { fixed }\left\{t_{i} \in T_{i}\right\} \backslash\{e\} .
$$

$R_{1}=\{\sigma \mid$ for some $i \in I,(i, \sigma) \in Q\} \cup\left\{t_{i}, t_{i} \theta_{i \alpha} \mid i \in I, t_{i} \in T_{i}, t_{i}\left(t_{i} \theta_{i \alpha}\right)^{-1} \neq e\right\}$.

By [5, Lemma 8, equation (20)], if $t_{i}\left(t_{i} \theta_{i \alpha}\right)^{-1} \neq e$, then $t_{i}\left(t_{i} \theta_{i \alpha}\right)^{-1}=$ $t_{j}\left(t_{j} \theta_{j \alpha}\right)^{-1}$ if and only if $t_{i}=t_{j}$. (This may also be proved by induction on the length of $t_{i}$.) This, together with the hypotheses of the theorem, implies that $R_{1}$ is finite.

Let $S$ be a finite subset of $G$ avoiding $H$. Adjoin to $R_{1}$ the identity $e$ and the representatives in $T_{\alpha}$ of the cosets contained in $H S$, together with all initial segments of the resulting set, to form $R_{2}$, still finite. (If $g=a_{i_{1}} \cdots a_{i_{n}}$ is in reduced 
form in $\prod^{*} A_{i}$, then all elements $a_{i_{1}} \cdots a_{i_{r}}(1 \leqq r \leqq n)$, and $e$, are called initial segments of $g$.) The inclusion of $e$ ensures that $R_{2}$ is not empty. Clearly $R_{2} \subseteq$ $\bigcup_{i \in I} T_{i}$ by property 2.2 .2 in the definition of uniform Schreier system.

For each pair $(i, \sigma)$ such that $\sigma \in R_{2} \cap S_{i}$, we define the sets

and

$$
X(i, \sigma)=\left\{a_{i} \mid a_{i} \in A_{i}, \sigma a_{i} \in R_{2}\right\}
$$

We then have

$$
Y(i, \sigma)=X(i, \sigma) X(i, \sigma)^{-1} \backslash\{e\} .
$$

$$
Y(i, \sigma) \cap B(i, \sigma)=\phi
$$

since by 2.2 .3 (i), $X(i, \sigma)$ is a subset of the transversal $Z(i, \sigma)$ for $B(i, \sigma)$ in $A_{i}$. Further since $R_{2}$ is finite, clearly so is $X(i, \sigma)$ and therefore also $Y(i, \sigma)$. Consider those pairs $(i, \sigma), \sigma \in R_{2} \cap S_{i}$, for which $Y(i, \sigma)$ is non-empty. By 2.3.1 and hypothesis, $B(i, \sigma)$ is contained in a subgroup $B_{1}(i, \sigma)$ say, of finite index in $A_{i}$ and avoiding $Y(i, \sigma)$. Thus $X(i, \sigma)$ can be extended to $Z_{1}(i, \sigma)$, a (finite) transversal for $B_{1}(i, \sigma)$ in $A_{i}$. For the pairs $(i, \sigma), \sigma \in R_{2} \cap S_{i}$, such that $Y(i, \sigma)=\phi$, define $B_{1}(i, \sigma)=A_{i}$ and $Z_{1}(i, \sigma)=\{e\}$. For every pair $(i, \sigma)$ with $\sigma \in R_{2} \cap S_{i}$, adjoin to $R_{2}$ all elements of $\sigma Z_{1}(i, \sigma)$ to obtain finally $R$. The subset $R$ is finite since the finiteness of $R_{2}$ implies that there exist only finitely many pairs $(i, \sigma)$ for which $Y(i, \sigma)$ is non-empty.

We shall now choose a uniform Schreier system

$$
\left(\left\{R_{i}, S_{i}^{\prime} \mid i \in I\right\},\left\{B_{1}(i, \sigma), Z_{1}(i, \sigma) \mid i \in I, \sigma \in S_{i}^{\prime}\right\},\left\{\theta_{i j}^{\prime} \mid i, j \in I\right\}\right)
$$

in $G$, such that $\bigcup_{i \in I} R_{i}=R$. Set

$$
R_{i}=\left(T_{i} \cap R_{2}\right) \cup\left(R \backslash R_{2}\right) .
$$

Then $S_{i}^{\prime}$ is defined in accordance with 2.2 .3 as the subset of those elements of $R_{i}$ which do not end in an element of $A_{i}$. For those pairs $(i, \sigma)$ with $\sigma \in S_{i}^{\prime} \cap R_{2}$ $\left(=S_{i} \cap R_{2}\right), B_{1}(i, \sigma)$ and $Z_{1}(i, \sigma)$ have been defined above. For those $(i, \sigma)$ with $\sigma \in S_{i} \backslash R_{2}$, define $B_{1}(i, \sigma)=A_{i}$ and $Z_{1}(i, \sigma)=\{e\}$.

Define $\theta_{i j}^{\prime}$ to agree with $\theta_{i j}$ on $T_{i} \cap R_{2}$ and as the identity on $R \backslash R_{2}$. That this definition of $\left\{\theta_{i j}^{\prime}\right\}$ is possible and satisfies 2.2.4, follows from the fact that

$$
\left(T_{i} \cap R_{2}\right) \theta_{i j}=T_{j} \cap R_{2} \quad \text { for all } i, j \in I .
$$

This is established as follows. Let $t_{i} \in T_{i} \cap R_{2}$. If $t_{i} \theta_{i j}=t_{i}$, then $t_{i} \theta_{i j}=t_{i} \in T_{j} \cap R_{2}$. Suppose on the other hand that $t_{i} \theta_{i j}=t_{j} \neq t_{i}$; then at least one of $t_{i}, t_{j}$ differs from $t_{i} \theta_{i \alpha}=t_{i} \theta_{i j} \theta_{j \alpha}=t_{j} \theta_{j \alpha}$. Thus at least one of $t_{i}\left(t_{i} \theta_{i \alpha}\right)^{-1}$ and $t_{j}\left(t_{j} \theta_{j \alpha}\right)^{-1}$ is non-trivial, whence by the definition of $R_{1} \subseteq R_{2}$, we have $t_{i} \theta_{i \alpha}\left(=t_{j} \theta_{j \alpha}\right) \in R_{1}$, and $t_{i}$ and $t_{j}$ must both belong to $R_{1}$. For if they both differ from $t_{i} \theta_{i \alpha}$, the definition of $R_{1}$ forces them to be in $R_{1}$, while if either equals $t_{i} \theta_{i x}$, it is trivially in $R_{1}$. Thus $\left(T_{i} \cap R_{2}\right) \theta_{i j} \subseteq T_{j} \cap R_{2}$, and 3.1.3 follows by symmetry since $\left(T_{j} \cap R_{2}\right) \theta_{i j}^{-1}$ $=\left(T_{j} \cap R_{2}\right) \theta_{j i}$. 
Secondly we verify that $R_{i}$ satisfies 2.2 .2 . Thus suppose $t \in \bigcup R_{i}$ ends in an element $a_{i} \in A_{i}$. If $t \in R_{2}$, then since $R_{2}$ is closed under taking initial segments, also $t a_{i}^{-1} \in R_{2}$. But $R_{2} \subseteq \bigcup T_{i}$. Thus, since $\left\{T_{i}\right\}$ satisfies 2.2.2, both $t$ and $t a_{i}^{-1}$ are in $T_{i}$. Hence $t, t a_{i}^{-1} \in T_{i} \cap R_{2} \subseteq R_{i}$. Suppose on the other hand $t \notin R_{2}$ : then $t \in R \backslash R_{2}$. It follows from the definition of $R$ that $t \in \sigma Z_{1}(j, \sigma)$ for some element $\sigma$ in $S_{j} \cap R_{2}$. Clearly, since $t \notin R_{2}$ and $t$ ends in $a_{i} \in A_{i}$, we must have $j=i$, $a_{i} \in Z_{1}(i, \sigma)$ and $t a_{i}^{-1}=\sigma$. Hence $t a_{i}^{-1} \in R_{2} \cap T_{i} \subseteq R_{i}$. Note that since $e \in T_{i} \cap R_{2}$, we have $e \in R_{i}$ for all $i$.

There only remains to check that condition 2.2.3 (i) is satisfied; i.e. that

$$
\sigma Z_{1}(i, \sigma)=R_{i} \cap \sigma A_{i}
$$

for each pair $(i, \sigma)$ with $\sigma \in S_{i}^{\prime}$. If $\sigma \in R_{2}$, then $\sigma Z_{1}(i, \sigma) \subseteq R_{i}$ by definition, whence $\sigma Z_{1}(i, \sigma) \subseteq R_{i} \cap \sigma A_{i}$. If $\sigma \notin R_{2}$, then by definition, $Z_{1}(i, \sigma)=\{e\}$ and $\sigma Z_{1}(i, \sigma)$ $=\{\sigma\} \subseteq R_{i} \cap \sigma A_{i}$. It remains to prove that $\sigma Z_{1}(i, \sigma) \supseteq R_{i} \cap \sigma A_{i}$. Let $x \in R_{i}$ $\cap \sigma A_{i}$; say $x=\sigma a_{i}$. If $\sigma \in R_{2}$, then by construction of $R, \sigma a_{i} \in \sigma Z_{1}(i, \sigma)$. If $\sigma \notin R_{2}$, then $\sigma a_{i} \notin R_{2}$ since $R_{2}$ is closed under taking initial segments, and therefore $\sigma a_{i} \in \sigma^{\prime} Z_{1}\left(j, \sigma^{\prime}\right)$ for some $\sigma^{\prime} \in R_{2} \cap S_{j}$ where $j \neq i$. It follows that $a_{i}=e$, and then trivially $x=\sigma \in \sigma Z_{1}(i, \sigma)$ since $e \in Z_{1}(i, \sigma)$. This completes the verification that 3.1.1 is a uniform Schreier system.

Let $H_{1}$ be the subgroup determined by this system in accordance with Theorem 2.4. Since $R_{i}$ is finite, $H_{1}$ has finite index in $G$. We now show that $H_{1} \geqq H$. To this end let $(i, \sigma)$ be an arbitrary element of $Q$ : then $\sigma \in R_{2}$ by definition of $R_{2}$, whence $\sigma \in T_{i} \cap R_{2} \subseteq R_{i}$, showing that $\sigma \in S_{i}^{\prime}$. Now for each $(i, \sigma) \in Q, B_{1}(i, \sigma)$ was chosen to contain $B(i, \sigma)$. We therefore have, for each $(i, \sigma) \in Q, \sigma B(i, \sigma) \sigma^{-1} \leqq$ $\sigma B_{1}(i, \sigma) \sigma^{-1}$. But the latter is a free factor of $H_{1}$ by Theorem 2.4, whence

$$
\sigma B(i, \sigma) \sigma^{-1} \leqq H_{1} .
$$

Define

$$
F_{1}=\operatorname{sgp}\left\{t_{i}\left(t_{i} \theta_{i \alpha}^{\prime}\right)^{-1} \mid i, \alpha \in I, \alpha \text { fixed as before, } t_{i} \in R_{i}\right\} .
$$

We shall show that

$$
F_{1}=F \text {. }
$$

Now $F=\operatorname{sgp}\left\{t_{i}\left(t_{i} \theta_{i x}\right)^{-1} \mid i, \alpha \in I, \alpha\right.$ as above, $\left.t_{i} \in T_{i}\right\}$. By definition of $R_{1}$, for $t_{i} \in T_{i}, t_{i}\left(t_{i} \theta_{i \alpha}\right)^{-1} \neq e$ only if $t_{i} \in R_{1}$; i.e. only if $t_{i} \in R_{1} \cap T_{i} \subseteq R_{2} \cap T_{i}$. Since $\theta_{i \alpha}^{\prime}$ agrees with $\theta_{i \alpha}$ on $R_{2} \cap T_{i}$ and $\left(R_{2} \cap T_{i}\right) \theta_{i \alpha}=R_{2} \cap T_{\alpha}$ by 3.1.3, we have $F_{1} \geqq F$. On the other hand if $t_{i} \in R_{i}$ is such that $t_{i}\left(t_{i} \theta_{i \alpha}^{\prime}\right)^{-1} \neq e$, then by the definition (3.1.2) of $R_{i}$, and that of $\theta_{i j}^{\prime}, t_{i} \in T_{i} \cap R_{1}$. It follows that $F_{1} \leqq F$, and 3.1.5 is proved.

We infer from 3.1.4, 3.1.5 and Theorem 2.4 that $H \leqq H_{1}$.

Next we prove that $H_{1}$ avoids $S$. By the definition of $R_{2}$, for each $s \in S$ there is a non-trivial element $r \in R_{2}$ such that $\operatorname{sr}^{-1} \in H$. Suppose $s \in H_{1}$ : then since 
$H \leqq H_{1}, r \in H_{1}$. However $r$ is a non-trivial member of a right transversal (at least one of the $R_{i}$ ) containing $e$. Hence $s \notin H_{1}$ and we have proved that $G$ is $H$-residually finite.

The second statement of the theorem is proved as follows. By Lemma 2.5 and the hypotheses of the theorem, for each pair $(i, \sigma) \in Q$ there exists a subgroup of $A_{i}$, say $D(i, \sigma)$, avoiding $Y(i, \sigma)$ and containing $B(i, \sigma)$ as a free factor. For these $(i, \sigma)$ we may therefore choose $B_{1}(i, \sigma)=D(i, \sigma)$. We have, by Theorem 2.4,

whereas

$$
H_{1}=F * \prod_{i \in I}^{*} \prod_{\sigma \in S_{i}}^{*} \sigma B_{1}(i, \sigma) \sigma^{-1}
$$

$$
H=F * \prod_{i \in I}^{*} \prod_{\sigma \in M}^{*} \sigma B(i, \sigma) \sigma^{-1}
$$

where $M_{i}=\{\sigma \mid(i, \sigma) \in Q\}$. However $M_{i} \subseteq S_{i}^{\prime}$ by the definition of $R_{i}$ and $S_{i}^{\prime}$, and since $\sigma B(i, \sigma) \sigma^{-1}$ is a free factor of $\sigma B_{1}(i, \sigma) \sigma^{-1}$ for $(i, \sigma) \in Q$, it follows that $H$ is a free factor of $H_{1}$. This completes the proof of the theorem.

\section{References}

[1] R. G. Burns, 'A note on free groups', Proc. Amer. Math. Soc., 23 (1969), 14-17.

[1] I. M. S. Dey, 'Schreier systems in free products', Proc. Glasgow Math. Assoc., 7 (1965-66), $61-79$.

[3] K. W. Gruenberg, 'Residual properties of infinite soluble groups', Proc. London Math. Soc. (3) 7 (1957), 29-62.

[4] M. Hall, Jr., 'Coset representation in free groups', Trans. Amer. Math. Soc. 67 (1949), $421-$ 432.

[5] S. MacLane, 'A proof of the subgroup theorem for free products', Mathematika 5 (1958), $161-183$.

[6] W. Magnus, A. Karrass and D. Solitar, Combinatorial group theory (Interscience, New York, 1966).

[7] A. Karrass and D. Solitar, 'On finitely generated subgroups of a free group', Proc. Amer. Math. Soc., 22 (1969), 209-213. 